Originalveröffentlichung in: Speer, Andreas ; Jeschke, Thomas (Hrsgg.): Schüler und Meister, Berlin 2016, S. 745-759 (Miscellanea Mediaevalia ; 39)

\title{
Der Architekt ohne Ausbildung als Normalfall in der italienischen Renaissance
}

\author{
Hubertus GüNTHER (München)
}

\section{Gesellschaftliche Bedeutung des Architekten}

Zunächst sei mit einigen Beispielen daran erinnert, wie wichtig für die Gesellschaft zu Beginn der Neuzeit die Arbeit derjenigen war, die große Bauprojekte betreuten, wie groß ihre Verantwortung war und wie notwendig es war, dass sie über hohe Qualitäten verfügten, um ihre Aufgaben zu meistern.

Die Renaissance der Architektur setzte in Florenz ein, damals zusammen mit Venedig und Mailand eine der größten und reichsten Städte des Abendlands. Der Dom hat fast die Ausdehnung einer kleinen mittelalterlichen Stadt, er überragt weit die übrigen Bauten um ihn herum, zudem besteht er aus kostbarem Material. Ein ganzes Quartier musste abgebrochen werden, um Platz für den neuen Chor zu schaffen. Insgesamt dürfte der Dom ungefähr so viel Geld gekostet haben wie damals alle Häuser in Florenz zusammen. Im Jahr 1296 war der Bau begonnen worden. Als der Tambour über der Vierung fertiggestellt War, stockten die Arbeiten lange Zeit. Man wusste nicht, wie die geplante Kuppel realisiert werden sollte ${ }^{1}$. So etwas wie sie hatte es noch nie gegeben. Die aufwendigste Kuppel aller Zeiten sollte entstehen, im Durchmesser so weit wie diejenige des Pantheons, aber in der luftigen Höhe von rund 50 Meter ansetzend. 1418 nahm die Dombauhütte endlich das Problem in Angriff. Sie rief Architekten aus dem ganzen Abendland zusammen um zu beraten, wie der Bau z $\mathrm{b}$ bewältigen war. Auch von diesen Experten wusste keiner, wie man vorgehen sollte. Man stelle sich nur vor, wie schmählich es für die aufstrebende Finanzmetropole gewesen wäre, wenn der Koloss ein Torso geblieben wäre. Da präsentierte Filippo Brunelleschi auf einmal ganz neue Ideen für eine Lösung. Er wurde daraufhin zum Dombaumeister berufen, und er rechtfertigte das in ihn gesetzte Vertrauen. Unter seiner Leitung gelang das Werk. Dieser Vorgang, sagt man allgemein, bezeichnet den Beginn der Neuzeit in der Architektur.

Im Jahr 1450 kam Francesco Sforza an die Macht über Mailand und beschloss Sogleich, sein Herzogtum im neuen Geist der Renaissance zu reformieren. Er stellte sich als idealer Fürst dar, um seine Herrschaft, die nicht allseits anerkannt

\footnotetext{
H. Saalmann, Filippo Brunelleschi: The Cupola of Santa Maria del Fiore, London 1980. Hier und in den folgenden Anmerkungen ist die Angabe von Literatur auf das beschränkt, was für den Beitrag grundlegend ist.
} 
war, zu festigen. Ein wesentlicher Teil seiner Politik bestand darin, die vielen verstreuten sozialen Einrichtungen der Lombardei zur Steigerung der Effizienz in einer Institution zusammenzuführen. Dafür sollte ein zentrales Hospiz in Mailand entstehen ${ }^{2}$. Er bat Piero de' Medici, einen Architekten aus Florenz für die Realisierung seiner Absicht zu nennen. Piero empfahl Antonio Averlino gen. il Filarete, und der begann dann das Hospiz zu errichten, das heute als Ospedale Maggiore bekannt ist. Hospize - Heime für Bedürftige aller Art, für Kranke, Waisen, Mittellose, Reisende etc. - bildeten überall Markenzeichen für Wohlstand und Gemeinsinn einer Stadt. Aber Francescos Projekt überbot bei Weitem alles der Art, was es bisher gegeben hatte. Es sollte sich auszeichnen durch riesige Dimensionen und schöne Gestaltung, Effizienz und Komfort. Es schloss Werkstätten und Industrieanlagen mit vielen innovativen technischen Einrichtungen ein. Es lag an einem Kanal, der ebenso zur Müllentsorgung wie zum Antreiben von Maschinen durch Wasserkraft diente. Dahinter stand die originäre Idee, dass die Hilfsbedürftigen nicht einfach dahinvegetierten, sondern, so weit wie möglich, arbeiteten, damit sich das Hospiz finanziell selbst tragen konnte. Das Hospiz war ein Monument für den avantgardistischen Regierungsstil des neuen Herzogs. So half Filarete, die Politik zu verwirklichen. Er führte sich auch als politischer Ratgeber seines Herrn auf. Er erteilte ihm Ratschläge für weitere neuartige Einrichtungen: für eine Strafrechtsreform, bei der die Todesstrafe durch Arbeit in einem Gefängnis ersetzt werden sollte, dessen Funktion in mancher Hinsicht dem Hospiz nahe kam, und für eine Schule, in der alle Formen von Ausbildung gebündelt sein sollten.

Das Exil in Avignon, das Schisma und anderes hatten die Kirche zu Beginn der Renaissance gründlich zerrüttet (1309-1417). Allmählich bahnten sich protestantische Bewegungen an. Die Christen im Osten verloren ihre Selbstständigkeit, als die Osmanen Konstantinopel eroberten (1453). Das prominenteste Gotteshaus der Christenheit, die Basilika über dem Begräbnisort des Apostelfürsten im Vatikan, spiegelte den desolaten Zustand der Kirche wider ${ }^{3}$. Sie war heruntergekommen, tektonisch unsicher, beschädigt, verschmutzt. Ihre Architektur galt inzwischen als schlecht, kunstlos, achtlos aus Spolien zusammengesetzt. Der Bau war recht schäbig gegenüber manchen neuen Domen oder im Vergleich mit der Hagia Sophia, der christlichen Hauptkirche im Osten, die Mehmet der Eroberer zu allem Unglück auch noch in eine Moschee umwandelte. Als Papst Nikolaus V. 1451 begann, die Peterskirche zu erneuern, musste er sich gegen den Vorwurf verteidigen, dass in der Zeit der höchsten Not das Geld nicht

2 H. Günther, Society in Filarete's „Libro architettonico“ between Realism, Ideal, Science Fiction and Utopia, in: Arte Lombarda. Nuova Serie 155/1 (2009), 56-80. Id., Italian Hospitals of the Early Renaissance, in: K. Ottenheym/K. De Jonge/M. Chatenet (eds.), Public Buildings in Early Modern Europe (Architectura Moderna 9), Turnhout 2010, 385-396.

3 F. Graf Wolff Metternich/C. Thoenes, Die frühen St.-Peter-Entwürfe 1505-1514 (Römische Forschungen der Bibliotheca Hertziana 25), Tübingen 1987. H. Günther, „Als wäre die Peterskirche mutwillig in Flammen gesetzt". Zeitgenössische Kommentare zum Neubau der Peterskirche und ihre Maßstäbe, in: Münchner Jahrbuch der bildenden Kunst 47 (1997), 165-210. 
für äußerliche Repräsentation verschwendet werden dürfte. Er legte daraufhin ausführlich dar, wie wichtig eine solche Maßnahme gerade in der Notzeit war, um die Achtung vor der Kirche und den Glauben der Christenheit zu festigen. Trotzdem kamen die Arbeiten nach dem Tod Nikolaus' V. ein halbes Jahrhundert lang kaum voran und stagnierten schließlich ganz. 1505 begann ein völliger Neubau. Um endgültig durchzusetzen, dass er vollendet wird, wurde der Abbruch der alten Basilika ohne lange Rücksicht auf ehrwürdige Monumente durchgezogen und die ersten Teile des Neubaus in fliegender Eile aufgeführt. Wieder erhob sich ein Sturm der Empörung, so heftig, wird berichtet, als wenn die Kirche „mutwillig in Flammen gesetzt worden wäre“. Die Ablassgelder, mit denen die immensen Kosten für den Neubau in ganz Europa eingetrieben wurden, führten nördlich der Alpen zu breiten Protesten. Sie trugen wesentlich dazu bei, dass sich die Kirche bis auf den heutigen Tag spaltete. In dem Fall war der leitende Architekt, Donato Bramante, nicht nur für die Planung und Durchführung der Baumaßnahmen verantwortlich, man gab ihm sogar die Schuld an der Zerstörung der Konstantinischen Basilika. Es hieß, er sei es gewesen, der den Neubau überhaupt angeregt habe. So hoch wurde sein Einfluss auf die Kurie eingeschätzt.

Natürlich nahmen die Architekten, die für die großen Projekte verantwortlich Waren, eine führende gesellschaftliche Stellung ein ${ }^{4}$. Die Stadt Florenz setzte Brunelleschi ein Denkmal im Dom neben den großen Feldherren, die ihr gedient hatten. Der Humanist Antonio Manetti schrieb seinen Lebenslauf nieder ${ }^{5}$. Filarete, Bramante und andere ließen sich wie vornehme Herrschaften eigene Medaillen prägen. Manche Architekten, wie Raffael oder Gian Cristoforo Romano und andere, verkehrten mit prominenten Wissenschaftlern und Literaten ${ }^{6}$. Sie arbeiteten sogar manchmal eng miteinander zusammen, so etwa Filarete mit dem Gräzisten Francesco Filelfo und anderen; der König von Neapel ließ für Francesco di Giorgio eigens Vitruv vom Lateinischen ins Italienische übersetzen. Manche Architekten, wie Antonio da Sangallo, Raffaels Nachfolger an der Bauhütte von St. Peter, häuften große Vermögen an, zwar teilweise anscheinend durch Immobilienhandel, aber der konnte sich eben auch aus ihrem Beruf ergeben. Die fürstlichen Architekten gehörten zur engen Umgebung ihrer Herren. Filarete beriet Francesco Sforza nicht nur, sondern führte auch den Erbprinzen in die Architektur ein. Wie hoch man den Einfluss Bramantes auf den Papst einschätzte, wurde eben erwähnt. Als Bramante dem Herzog von Mailand diente, konnte er es sich leisten, für einige Zeit einfach zu verschwinden, ohne

${ }^{4}$ Im Folgenden stütze ich mich auf meine Beiträge über Beruf und Ausbildung des Architekten in der Renaissance in: R. Johannes (ed.), Entwerfen. Architektenausbildung in Europa von Vitruv bis Mitte des 20. Jahrhunderts. Geschichte, Theorie, Praxis, Hamburg 2009, 215-275, 743-768, und in: W. Nerdinger, Der Architekt. Geschichte und Gegenwart eines Berufsstandes, München-London-New York 2012, vol. 1, 59-80.

5 A. Manetti, Vita di Filippo Brunelleschi, edd. D. de Robertis/G. Tanturlis, Milano 1976.

${ }^{6}$ H. Günther, Das Studium der antiken Architektur in den Zeichnungen der Hochrenaissance, Tübingen 1988. 
entlassen zu werden; der Herzog bemühte sich vielmehr, ihn in Rom und Florenz zu suchen. Raffael, Bramantes Nachfolger als Architekt der Peterskirche, hat selbst berichtet, dass er jeden Tag mit dem Papst den Neubau der Peterskitche besprach ${ }^{7}$. Als Michelangelo, Antonio da Sangallos Nachfolger als Architekt der Peterskirche, gestorben war, ließ der Herzog von Florenz seinen Leichnam in einer Nacht- und Nebelaktion aus Rom rauben und veranlasste, ihm ein pompöses Begräbnis wie einem Fürsten auszurichten.

\section{Aufgaben des Architekten}

In der einschlägigen Literatur der Renaissance wird derjenige als Architekt bezeichnet, der einen Bau konzipiert und die Bauarbeiten gewöhnlich leitet. Die Arbeiten führten Bauhandwerker verschiedener Sparten aus. Wir halten uns hier an diese Unterscheidung, weil sie zutrifft für die Architekten, die berühmt geworden sind und die Kunstgeschichte hauptsächlich beschäftigen. In der Praxis wurde allerdings sprachlich nicht so klar zwischen den beiden Berufsständen geschieden. Die Baudokumente gebrauchen die Begriffe „Architekt“, „Ingenieur", „Steinmetz" etc. oft synonym, und in jedem Einzelfall ist fraglich, was einer, der so angesprochen wurde, wirklich leistete.

Ruhm und Glanz des Architektenberufs verbanden sich mit dem Entwurf und der künstlerischen Idee. Aber die Architekten kümmerten sich keineswegs nur um die formale Gestaltung. Sie waren auch verantwortlich für die praktische Seite des Baubetriebs. Nur ist dieser Aspekt nicht so spektakulär, und deshalb gibt es wenige Zeugnisse dafür. Auch wenn die Leiter eines Baus nicht die praktischen Aufgaben selbst erledigten, mussten sie wenigstens kontrollieren können, was geschah.

Im Vorfeld der Planung war es oft nötig, Vermessungen durchzuführen. Geräte dafür, Lehrbücher und Skizzen zur ersten Fixierung der Ergebnisse sind aus der Renaissance erhalten. Dann musste das Baumaterial ausgesucht, seine Menge berechnet und schließlich die Kosten veranschlagt werden. Auch solche Kostenvoranschläge sind erhalten. Filarete oder Vincenzo Scamozzi haben beschrieben, wie eine solche Kalkulation aussehen soll ${ }^{8}$. Für den Neubau der Stadt Pienza berichtet der Bauherr, Papst Pius II., selbst ausdrücklich, dass ihm der Architekt den Kostenvoranschlag vorgelegt hatte ${ }^{9}$. In dem Fall war, wie es auch jetzt noch gelegentlich geschieht, der Aufwand viel zu niedrig angesetzt worden. 7 J. Shearman, Raphael in Early Modern Sources (1483-1602), 2 vols., New Haven-London
2003, vol. 1, 180-183.

8 A. Averlino detto il Filarete, Trattato di architettura, 4. Buch, edd. A. M. Finoli/L. Grassi, A. Averlino detto il Filarete, Trattato di architettura, 4. Buch, edd. A. M. Finoli/L. Ge 1615 ,
Milano 1972, 90-120. Vincenzo Scamozzi, L'idea della architettura universale, Venetiis
$77-83$.

${ }^{9}$ Pius II., Commentarii rerum memorabilium, ed. A. van Heck, 2 vols., Città del Vaticano 1984 , vol. 2, 533. I commentarii, 9, 25, ed. G. Bernetti, 5 vols., Siena 1972-76, vol. 3, 227 
Dann bestimmten die Architekten, wie die Fundamente angelegt werden sollten. Für Gian Lorenzo Bernini beispielsweise ist belegt, dass er sich persönlich um die sachgerechte Ausführung kümmerte: Er stieg in die Baugrube, die für die neue Stadtfront des Louvre ausgehoben war, um die Arbeiter zu belehren, wie sie die Fundamente mauern sollten ${ }^{10}$. Damit handelte er sich freilich nur den Widerwillen der Handwerker ein, denn er wollte ihnen beibringen, was er aus Italien her gewohnt war, während sie sich an das hielten, was in Paris üblich war.

Selbstverständlich wurde vorausgesetzt, dass der Architekt für die Tektonik der Bauten zuständig ist. Wenn Mängel in der Tektonik von Bauten auftraten, wurde er dafür verantwortlich gemacht. Beispielsweise gab man Bramante die Schuld daran, dass Risse in den Vierungspfeilern der Peterskirche auftraten, oder der Senat von Venedig warf den Stadtbaumeister Jacopo Sansovino ins Gefängnis zur Strafe dafür, dass unter seiner Bauleitung die Gewölbe der Biblioteca Marciana gegenüber dem Dogenpalast zusammenbrachen ${ }^{11}$, etc.

Der Bau der Kuppel des Florentiner Doms bildet ein Paradigma für die technischen Leistungen des leitenden Architekten. Die Form stand bereits seit mehr als einem halben Jahrhundert im Wesentlichen fest. Nur die Ausführung stellte technische und logistische Anforderungen von bisher ungekanntem Ausmaß. Brunelleschi hatte vor allem die Probleme zu lösen, welche Mauertechniken und Armierungen geeignet waren, den Halt des Riesenwerks zu sichern, mit welchen Gerüsten und Baumaschinen man in der luftigen Höhe arbeiten konnte, mit Welchen Kränen Material und Bauleute hochbefördert werden sollten. Auf dem Grabmal, das ihm die Stadt Florenz setzte, wird die Erfindung der Maschinen als seine großartigste Leistung hingestellt. Francesco di Giorgio, Leonardo da Vinci und andere haben viele Zeichnungen von Maschinen verschiedenster Art hinterlassen, besonders solcher zum Heben von schweren Gegenständen und von Wasser. Filarete entwickelte für das zentrale Hospiz von Mailand selbst die modernen technischen Einrichtungen. Federico da Montefeltre ließ am Herzogspalast von Urbino eine Serie von Reliefs anbringen, die Maschinen darstellen. Offenbar hielt er die Konstruktion solcher Geräte nicht für niedrige Arbeit, sondern schätzte ebenso wie die Stadt Florenz die intellektuelle Kapazität, die dahinter stand.

Die obersten Architekten eines Herrschers, einer Kommune oder des Papstes sollten sich nicht nur um die Repräsentationsbauten kümmern. Sie waren auch Zuständig für Stadtplanung oder die Anlage von Straßen und den Bau von Brücken, für Wasserbau wie die Anlage von Kanälen, Schleusen, Dämmen, Leitungen, Zisternen oder Brunnen, ebenso für öffentliche Nutzbauten wie Hospitäler,

\footnotetext{
${ }^{10}$ P. Fréart de Chantelou, Journal de voyage du Cavalier Bernin en France, ed. M. Stanic, Paris $2001,158$. D. Howard, Jacopo Sansovino. Architecture and Patronage in Renaissance Venice, New Haven-
London ${ }^{2} 1987,20$ sq.
} 
Schulen oder Gefängnisse ${ }^{12}$. Antonio da Sangallo hat den großen Brunnen der Stadt Orvieto errichtet; vom Sieneser Architekten Baldassare Peruzzi ist eine Serie sorgfältig angelegter und kommentierter Zeichnungen für die Anlage eines Staudamms der Bruna erhalten ${ }^{13}$. Zur Stadtplanung gehörten auch die Parzellierung von Grundstücken, die Kalkulation von Straßensteuern etc. Wenn auch nur sporadisch, so gibt es zumindest in Rom doch Zeugnisse genug dafür, dass sich die obersten Architekten auch um minimale Belange, die mit der Stadtplanung verbunden waren, kümmerten ${ }^{14}$.

Die obersten Architekten mussten sich auch um Stadtmauern, Festungen und Kriegsmaschinen kümmern. Das war ein wesentliches Gebiet. Als sich Leonardo da Vinci beim Herzog von Mailand um eine Anstellung bewarb, stellte er eine Liste seiner Fähigkeiten auf (um 1480). Sie beginnt mit der Konstruktion von Kampfmaschinen. Eine essentielle Aufgabe der Architekten bestand zu Beginn der Neuzeit darin, für die Befestigungen Formen zu entwickeln, die der neuerdings aufkommenden Artillerie und dem damit zusammenhängenden Wandel der Kriegsstrategie gewachsen waren. Man hatte zu berücksichtigen, wie eine Wehrmauer beschaffen sein muss, um den Druck von Kanonenkugeln auszuhatten, wie Kasematten angelegt sein sollten, wie man das Vorfeld der Mauern unter den neuen Bedingungen deckt, wie man eine Batterie effizient aufstellt etc. Der Florentiner Architekt Giuliano da Sangallo entwickelte die modernen Bastionen, die im Prinzip bis zum 18. Jahrhundert maßgeblich für den Wehrbau blieben. Dabei mögen ihn Militärexperten unterstützt haben, aber Vasari schreibt ausdrücklich ihm selbst die Erfindung einer besonderen Methode zur Dämpfung des Rückstoßes von Geschützen $\mathrm{zu}^{15}$.

\section{Anforderungsprofil des Architekten}

Der Architekt sollte also ein weites Spektrum an praktischen Fähigkeiten mitbringen. Er brauchte sachliches Wissen über Baumaterialien, Mauertechniken etc.; unabdingbar waren gute mathematische Kenntnisse für die Planung, für das Einmessen von Grund und Boden und von Bauteilen, für die Berechnung

12 H. Günther, Das Trivium vor Ponte S. Angelo. Ein Beitrag zur Urbanistik der Renaissance in Rom, in: Römisches Jahrbuch für Kunstgeschichte 21 (1984), 165-251, 197-207 (Die Verant wortlichen für die Stadtplanung der Renaissance in Rom und die Architekten von St. Peter).

13 H. Wurm, Baldassarre Peruzzi. Architekturzeichnungen, Tübingen 1984, 295-300. S. di Pasquale, Riflessioni e note sui progetti di Baldassarre Peruzzi per la formazione di un lago artificiale nel territorio di Siena, in: M. Forlani Conti (ed.), Rilievi di Fabbriche attribuite a Baldassarrc Peruzzi, Ausstellungskatalog, Siena 1982, 71-88.

${ }^{14}$ H. Günther, Antonio da Sangallo progetta una via a Roma: La sistemazione della via Agonale H. Günther, Antonio da Sangallo progetta una via a Roma: La sistemazione della via Arenitet-
presso piazza Navona, in: G. Alisio (ed.), I Disegni d'Archivio negli Studi di Storia dell'Aren
tura, Napoli 1994, 20-30. 15 G. Vasari, Le vite de' piu eccellenti pittori scultori ed architettori, ed. G. Milanesi, 9 vols., Firenze
$1878-1885$, vol. 4,269 . 
von Materialbedarf oder die Kalkulation von Baukosten. Es gibt genügend Zeugnisse dafür, dass die Architekten die Erwartungen im Allgemeinen erfüllten. Für den Bauentwurf waren nicht nur künstlerisches Ingenium und die Fähigkeit zum Planzeichnen nötig. Weil die Architektur damals als Wissenschaft bewertet wurde, erwartete man zudem die Beherrschung einer formalen Theorie, eine enzyklopädische Allgemeinbildung, gewisse literarische und rhetorische Qualitäten. Ein guter Architekt musste sich in der antiken Architektur auskennen und in der Lage sein, daraus Gesetzmäßigkeiten für seine eigene Planung abzuleiten. Das erforderte hohes intellektuelles Niveau. Man konnte sich noch nicht wie heute einfach auf Lehrbücher verlassen. Die antiquarischen Forschungen standen ganz am Anfang. Man musste selbst die Ruinen untersuchen und die einschlägigen antiken Schriften deuten. Es sind viele Studien von Architekten aus der Renaissance überliefert, die von der Erforschung der Ruinen und dem Bemühen zeugen, Vitruvs Architekturtraktat, das einzige erhaltene Architekturtraktat aus der Antike, zu verstehen ${ }^{16}$. Einige Architekten haben selbst Traktate über ihr Fach verfasst, andere haben sich als Dichter profiliert, so etwa Bramante. Wie nützlich eine gute Rhetorik für den Architekten sein konnte, um Aufträge zu erhalten und um seine Vorstellungen durchzusetzen, führt Manetti in seinem Bericht darüber vor Augen, wie Brunelleschi mit der Florentiner Domopera umging ${ }^{17}$.

Im Ganzen gingen die erwarteten Qualitäten über direkten praktischen Nutzen hinaus. Aber sie waren auch als Zeugnisse einer Grundlage für ein vernünftiges Urteilsvermögen gedacht, und sie wirkten letztlich als Werbung für den Architekten. Zum Abschluss sei noch ein Beispiel dafür angeführt, wie ein Architekt seinen weiten geistigen Horizont zur Schau stellte: Der Präfekt der Bauten des Herzogs von Mantua, Giovanni Battista Bertani baute sich beim Hafen Von Mantua ein Haus mit einem rückwerten Hof, der an den Hafen grenzte. Auf dem Hof richtete er eine Ziegelei ein, in der alle Arten von tönernen Bauelementen wie Ziegel, Dachschindeln, Leitungen etc. produziert wurden. Mit den Produkten handelte er. An der Straßenfassade seines Hauses brachte er Modelle an, die mit Inschriften von lateinischen Fachausdrücken und Texten erklären, Wie Vitruvs Beschreibung der ionischen Säulenordnungen zu verstehen ist; er publizierte dazu auch eine geschlossene Abhandlung im Druck, und die verkaufte er in seinem Haus ${ }^{18}$.

\section{Ausbildung des Architekten}

Nach all dem sollte man denken, dass die Architekten eine gediegene Ausbildung für ihren Beruf erhielten. Aber so war es nicht.

16

Günther, Studium (nt. 6). P. N. Pagliara, Vitruvio da testo a canone, in: S. Settis (ed.), Memoria dell'Antico nell'Arte italiana, 3 vols., Torino 1984-1986, vol. 3, 5-85.

${ }_{18}$ Manetti, Vita di Filippo Brunelleschi, edd. de Robertis/Tanturlis (nt. 5), 77-88.

18 H. Günther, Künstlerhäuser seit der Renaissance $1470-1800$, in: M. T. Brandlhuber/M. Buhrs (eds.), Im Tempel des Ich. Das Künstlerhaus als Gesamtkunstwerk. Europa und Amerika 18001948, Ausstellungskatalog, München 2013, 16-29. 
Brunelleschi, der die Lösung dafür fand, wie man die Kuppel des Florentiner Doms ausführen konnte, war von Beruf Goldschmied und hatte keine nennenswerte Erfahrung im Bauen, als er zum Dombaumeister berufen wurde. Der Domopera kamen dann doch Bedenken, ob er die schwierige Aufgabe allein bewältigen könne, und sie stellte ihm einen zweiten Architekten zur Seite: Das war Lorenzo Ghiberti, auch ein Goldschmied, gerade berühmt geworden durch die vergoldete Bronzetür im Hauptportal des Florentiner Baptisterium, die sogenannte Paradiestür. Aber mit Architektur hatte er bisher nichts zu tun.

Filarete, der das Ospedale Maggiore mit seinen innovativen technischen Einrichtungen baute, hatte ebenfalls keine Erfahrung im Baubetrieb, als ihn Piero de' Medici nach Mailand empfahl. Auch er war als Goldschmied ausgebildet. Er war, ähnlich wie Ghiberti, soeben dadurch berühmt geworden, dass er die versilberten Türflügel im Hauptportal der Peterskirche in Rom geschaffen hatte. Wir können noch die anderen Architekten durchgehen, die erwähnt wurden, und kommen zu ähnlichen Ergebnissen: Bramante und natürlich Raffael begannen ihre Karriere als Maler; Michelangelo und Bernini waren bekanntlich als Bildhauer ausgebildet; ebenso Jacopo Sansovino, der Stadtbaumeister der Republik Venedig, oder Bernardo Rossellino, der für Papst Pius II. Pienza baute.

Die Idee, die Dombaumeister von außerhalb des Baubetriebs zu berufen, hatte in Italien Tradition: Dem Bau des Florentiner Doms stand anfangs der Bildhauer Arnolfo di Cambio vor; 1334 wurde der Maler Giotto zur Bauleitung berufen, nach ihm der Bildhauer Andrea Pisano. Keiner von ihnen hatte Erfahrungen als Architekt. Als Begründung für die Anstellung des fast siebzigjährigen Giotto hieß es einfach, er sei mehr als irgendein anderer auf der Welt geeignet, und man hoffe, er werde sich in Florenz niederlassen, damit viele von der Kunst des hochberühmten Meisters lernen könnten ${ }^{19}$. Die Stadt Siena berief um die gleiche Zeit (1339) den Goldschmied Lando di Pietro zum Leiter ihrer Dombauhütte, obwohl er vordem ebenfalls nicht als Architekt tätig gewesen war.

Sogar Humanisten und Schriftsteller wurden als Architekten angestellt, so Leon Battista Alberti oder Fra Giocondo. Alberti verfasste auch ein enorm gelehrtes Architekturtraktat (1485 posthum erschienen), Fra Giocondo publizierte die für die Renaissance maßgebliche Vitruv-Edition (1511). Fra Giocondo baute in Paris und im Veneto Brücken, kümmerte sich für die Republik Venedig um Hafenanlagen und Verteidigungswerke und wurde nach Bramantes Tod $\mathrm{Zu}^{-}$ sammen mit Raffael zum Architekten der Peterskirche berufen. Übrigens waren viele von den Humanisten auch für die literarische Tätigkeit, die ihren Ruhm ausmachte, nicht ausgebildet. Alberti, der auch mehrere Traktate über soziale Themen und Bühnenwerke verfasste, hatte wie viele Humanisten Jura studiert. Auch Fürsten entwarfen eigenständig Bauten. So ist es für Kaiser Maximilian I. oder König Franz I. von Frankreich belegt ${ }^{20}$. Die ersten einschlägigen Traktate

19 Saalmann, Filippo Brunelleschi (nt. 1), 178 sq.

19 Saalmann, Filippo Brunelleschi (nt. 1), 178 sq.
Cf. die Beiträge von M. Chatenet und H. Günther in: A. Calzona (ed.), Il principe architetto,
Firenze 2002, 533-544, 493-516. 
über Architektur und Kriegskunst (dazu gehörten auch Konstruktion von Kriegsmaschinen und Wehrbau), die im Druck erschienen, waren in lateinischer Sprache verfasst (Alberti und Roberto Valturio ${ }^{21}$ ); sie richteten sich demnach wohl mehr an gelehrte Auftraggeber als an Künstler.

Eine spezielle Architektenausbildung war in Italien zu Beginn der Neuzeit überhaupt nicht vorgesehen. Es gab eine Ausbildung für Bauhandwerker wie Maurer, Steinmetze oder Zimmerleute. Aber aus diesen Handwerken gingen gewöhnlich nicht die Architekten großer Bauunternehmungen hervor. Sie waren als bildende Künstler und meistens als Goldschmiede ausgebildet. Die Künstler hatten mit Architekten gemein, dass sie künstlerische Begabung mitbrachten und Zeichnen gelernt hatten; man konnte nach damaligen Maximen auch einige theoretische Verbindungen zwischen Malerei und Architektur schlagen. So hebt Cennino Cennini in seinem Kunsttraktat (um 1400) hervor, dass die Architektur und ihre Glieder schöne Elemente der Malerei seien, und Alberti erwartet in seinem Architekturtraktat, dass die Behandlung der Säulenordnungen in erster Linie die Maler interessieren würde ${ }^{22}$; warum das so sein soll, bleibt im Dunkeln. Aber solche Gedanken reichen längst nicht aus um zu erklären, warum man bildende Künstler als Architekten einstellte. Noch weniger begründen sie, warum man für diese Aufgabe ausgerechnet Goldschmiede bevorzugte.

Die Ausbildung der großen Architekten in den Künstlerwerkstätten hing offenbar mit der sozialen Herkunft und der Organisation der Berufsverbände zusammen.

Die Architekten bzw. bildenden Künstler, die zu Ruhm gelangten, stammten auffällig oft aus gutbürgerlichen Familien: Beispielsweise die Väter von Brunelleschi, Leonardo oder Michelangelo oder Ghibertis Großvater waren Notare. Viele Von den Künstlern, die zu Ruhm gelangten, sollten ursprünglich gehobene Berufe ergreifen: Brunelleschi und andere sollten Jura studieren, Jacopo Sansovino War für den Beruf des Kaufmanns bestimmt etc. Es gibt eine Ausnahme, die zu berühmt ist, um hier ganz übergangen zu werden: Andrea Palladio, der einzige Architekt, der einer ganzen Stilrichtung den Namen gegeben hat: dem Palladianismus. Aber die Ausnahme bestätigt nur die Regel. Palladios Aufstieg fand unter einzigartigen Bedingungen statt. Ein großer Schriftsteller, der Graf Giangiorgio Trissino, entdeckte den jungen Steinmetzen, sorgte für seine Bildung, reiste mit ihm viermal nach Rom, damit er die Antike studieren und anschlieBend sogar Rom-Führer verfassen konnte, und reichte ihn dann im venetischen Hochadel herum, sodass er Bauaufträge erhielt.

Die sozialen Bedingungen der Herkunft wirkten sich auf die Qualität der Ausbildung aus. Welche Grundausbildung die Künstler als Kinder erhielten, ist $k_{\text {aum }}$ je individuell überliefert. Aber wahrscheinlich war sie so gut, wie es dem

\footnotetext{
${ }_{22}^{21}$ L. B. Alberti, De re aedificatoria, Florentiae 1485; R. Valturio, De re militari, Veronae 1472.

${ }^{2}$ C. Cennini, Il libro dell'arte, cap. 87, ed. F. Brunello, Vicenza 1971, 96. L. B. Alberti, L'Architettura (De re aedificatoria). Testo latino e traduzione a cura di G. Orlandi (Trattati di architettura 1), Milano 1966, 529 (Anfang 7. Buch).
} 
sozialen Stand ihrer Familien, den intellektuellen Ansprüchen der für sie vorgesehenen Berufe und der Qualität der Werkstätten entsprach, in die sie danach eintreten sollten. Über die gutbürgerliche Grundausbildung generell gibt es genügend Nachrichten.

Der Aufstieg von Wissenschaft und Bildung in der Renaissance war von einem sprunghaften Ausbau des Schulwesens begleitet. Italien stand an der Spitze der Entwicklung. Es gab gewöhnlich drei Arten von Schulen: Elementarschulen, in denen Kinder Lesen und Schreiben übten; Abakusschulen, die Rechnen und geometrische Grundregeln beibrachten; Grammatikschulen, die Latein als internationales Verständigungsmittel lehrten und über die antiken Schriften historische Kenntnisse und allgemein geistige Bildung vermittelten. Von Brunelleschi berichtet Antonio Manetti: Er erhielt in der Jugend Unterricht im Lesen, Schreiben und Rechnen ${ }^{23}$. So sei es üblich für die „,huomini da bene“ in Florenz. Überdies habe er "qualche lettere" gelernt, er besuchte also eine Grammatikschule. Sonst sei es unüblich, „lettere" zu lernen, wenn man nicht dazu bestimmt sei, Arzt, Jurist oder Priester zu werden.

Anscheinend wurde in manchen Schulen auch Zeichnen gelehrt. Über die musische Ausbildung konnte zudem die Architektur in die Grundausbildung einbezogen werden. Matteo Palmieri schreibt in seinem Traktat über das gesellschaftliche Leben (1465): Die Kinder sollten an alle Wissenschaften herangeführt werden und einige Kunstfertigkeiten üben, zusätzlich zu Musizieren, Malen und Schnitzen auch "gute Bauten erfinden“" (,imaginare degni edifici“ ${ }^{\circ 24}$.

Die Handwerker traten durchschnittlich mit vierzehn Jahren für vier Jahre als Lehrling in eine Werkstatt ein. Die Auswahl der Werkstatt war nicht nur von der Branche, sondern auch von sozialen Bedingungen geleitet, und diese waren in Italien mit dem Zunftwesen verbunden.

Bildende Künstler und Bauhandwerker gehörten zu Zünften unterschiedlichen Standes. In Florenz waren die Zünfte in zwei Klassen geteilt: die oberen, die Arti maggiori, und die niederen, die Arti minori. Die Arti maggiori repräsentierten allein die Bürgerschaft im Stadtrat, die Arti minori hatten dort keine Stimme. Die Bauhandwerker gehörten zu den Arti minori. Sie bildeten eine eigene Zunft: die Arte dei Maestri di Pietra e Legname. Maler und Goldschmiede gehörten dagegen zu den Arti maggiori. Die Maler waren der Zunft der Medici e Speciali, der Ärzte und Apotheker, angeschlossen. Die Goldschmiede waren am besten gestellt. Sie waren mit reichen Fabrikanten und Kaufleuten zusammengeschlossen. Aus sozialer Warte ist leicht einzusehen, warum die Goldschmiede die höchste gesellschaftliche Stellung unter den Künstlern einnahmen. Sie verarbeiteten kostbares Material, Edelmetalle und Edelsteine, und handelten damit. Bei ihnen kam das meiste Geld zusammen. Sie brauchten besonders gute Eigenschaften: viel Sachkenntnis, um ihr Material zu beurteilen, viel Verstand, um gut zu kalkulieren, gute Umgangsformen, um ihre wohl situierte Klientel

23 Manetti, Vita di Filippo Brunelleschi, edd. de Robertis/Tanturlis (nt. 5), 39.

${ }^{24}$ M. Palmieri, La vita civile, ed. G. Belloni, Firenze 1982, 39. 
seriös zu bedienen. Daher war das Gewerbe schon im Mittelalter hoch angesehen. Von ihm ging lange vor der Renaissance der Brauch aus, Kunstwerke zu signieren. Das gilt besonders für aufwendige Bronzeportale. Anscheinend weil sie so kostbar waren, brachten die Künstler an ihnen ihr Firmenzeichen an.

Die Wahl der Zunft war nicht an Berufssparten gebunden. Maler konnten bei den Goldschmieden eingeschrieben sein und umgekehrt. Auch Bürger, die kein Handwerk ausübten, durften einer Zunft beitreten. Nur auf diese Weise konnten sie in den Stadtrat kommen. So finden sich in den höheren Zünften von Florenz illustre Humanisten oder Literaten wie Dante (bei den Ärzten). Wegen des hohen sozialen Ansehens gaben Väter aus gutgestellten Familien ihre Söhne, wenn sie Künstler werden wollten, gern zu Goldschmieden in die Lehre. Die Entscheidung hing weniger davon ab, in welcher Kunstgattung die Jungen später hauptsächlich hervortraten. Giorgio Vasari stellt in der ,Vita' des berühmten Malers Sandro Botticelli fest: „Damals bestand eine enge Vertrautheit und eine geradezu beständige Verbindung zwischen Goldschmieden und Malern"25.

Die Zünfte achteten hauptsächlich auf den Geschäftsverkehr. Ihre Statuten waren rein wirtschaftlich ausgerichtet.

In vielen italienischen Städten, so auch in Florenz, kannten die Zünfte keine klare Abgrenzung zwischen Lehrling, Geselle und Meister nach Ausbildungsstand. Der Meister zeichnete sich einfach dadurch aus, dass er eine eigene Werkstatt führte. Um sich selbständig zu machen, brauchte er nur das nötige Kapital, eine Klientel und Erfahrung. Es war nicht nötig und nicht einmal möglich, dafür eine Prüfung zu absolvieren. Es gab auch keine Gesellenprüfung. Lehrlinge und Gesellen hatten beide den Status bezahlter Arbeiter.

Die guten Meister achteten offenbar von sich aus auf das Ansehen ihrer Werkstatt und die Qualität ihrer Arbeit. Es gab keine Normen dafür, was der Meister lehren sollte. Es wird auch kaum etwas konkret darüber berichtet, was in den Künstlerwerkstätten gelehrt wurde und wie die Ausbildung ablief ${ }^{26}$. Im Kunsttraktat des Cennino Cennini ist ein Absatz der Ausbildung der Maler gewidmet ${ }^{27}$. Dort heißt es, Lehrlinge sollen die praktischen Vorarbeiten lernen, die für die Malerei nötig sind, wie Farben mischen, Leim bereiten, grundieren etc. Dann sollen Lehrlinge und Gesellen unablässig zeichnen. Am Beginn des Zeichnens stand das Kopieren von Werken des eigenen Meisters oder älterer Meister. Davon zeugen etwa Michelangelos Kopien nach Giotto, Masaccio und Schongauer. Erst als Geselle sollte man beginnen, selbst mit Farben zu malen. Aus der ganzen einschlägigen theoretischen Literatur der Renaissance geht nicht wesentlich mehr zu dem Thema hervor.

Die weitere Ausbildung wird von der Qualität und vom Wirkungsfeld des Meisters abgehangen haben. Mit der Wahl der Werkstatt war wohl oft der spätere

\footnotetext{
${ }^{25}$ Vasari, Le vite, ed. Milanesi (nt. 15), vol. 3, 310.

${ }^{26}$ F. Ames-Lewis, The Intellectual Life of the Early Renaissance Artist, New Haven-London 2002, 17-60.

27. Cennini, Il libro dell'arte, cap. 104, ed. Brunello (nt. 22), 109 sq.
} 
Weg des Lehrlings vorbestimmt. Davon hing ab, welcher geistige Horizont sich ihm eröffnete. Die Qualität des Meisters schlug sich bei den geeigneten Schülern nieder. Umgekehrt werden die exquisiten Meister von ihren besseren Lehrlingen eine gediegene Schulausbildung erwartet haben, damit sie präpariert waren, das Lehrangebot aufzunehmen, das ihnen geboten wurde. In einer Werkstatt wie etwa derjenigen Giovanni Bellinis, die viele später berühmte Maler hervorbrachte, werden die Schüler zudem durch Konkurrenz untereinander gereift sein. Eine Statistik dürfte ergeben, dass die berühmt gewordenen Architekten und bildenden Künstler der Renaissance mehrheitlich in Werkstätten berühmter Künstler ausgebildet wurden, so etwa Leonardo, Raffael, Tizian oder Michelangelo. Michelangelo trat, obwohl er Bildhauer werden wollte, in die Werkstatt des Domenico Ghirlandaio ein, der zwar Maler war, aber die größten Aufträge für Fresken in Florenz erhielt.

Die Lernprozesse, die über das Lehrangebot hinausgingen, beruhten auf Eigeninitiative. Nach dem, was die wenigen Quellen dazu - Benvenuto Cellini über sich selbst oder Ascanio Condivi und Giorgio Vasari über Michelangelo berichten, begannen die Jungen schon während ihrer Lehrzeit, sich selbstständig auszubilden ${ }^{28}$. Besonders die bildenden Künstler, die sich für Architektur interessierten, mussten sich wohl eigenständig fortbilden. Ebenso eigenständig bilden mussten sich Leute aus anderen Berufen, die es in die gleiche Richtung drängte. Für das Studium der antiken Bauten, das zur eigenständigen Ausbildung gehörte, übersiedelten etliche junge Künstler sogar für einige Jahre nach Rom. Da schlugen sie sich dann als Hilfskräfte durch, wie es etwa für Cronaca oder Vignola überliefert ist, andere wie Vincenzo Scamozzi oder Palladio erhielten von ihrem Elternhaus oder einem begüterten Mäzen finanzielle Unterstützung.

Die Erfahrung eigenständig zu lernen, blieb auch für Fortgeschrittene bestimmend. Davon zeugen die zahllosen Neuerungen, die in der Renaissance aufkamen, wie Brunelleschis Experiment zur Perspektiv-Konstruktion oder Paolo Uccellos Kampf mit der Perspektive und natürlich besonders Leonardos wegweisende Studien in viele Richtungen. Leonardo, Michelangelo, Cellini und andere haben betont, wie viel sie im Alleingang lernten.

Die meisten Architekten gelangten wohl im Lauf ihrer Tätigkeit durch den Rat von Handwerksmeistern zu einem Einblick in die Baupraxis. Zur eigenen Fortbildung gehörte eben auch, auf Erfahrenere zu hören. Darüber wird sehr wenig konkret berichtet. Raffael bezeugt selbst, dass der Papst ihm Fra Giocondo als Architekt der Peterskirche an die Seite stellte, damit er von ihm lernen könne ${ }^{29}$. Cellini scheibt generell: „Es ist immer lehrreich, aus der Erfahrung anderer Nutzen zu ziehen", und schildert sehr anschaulich, wie er auf den Rat von Handwerkern angewiesen war, als er erstmals einen großen Bronzeguss

28 B. Cellini, La vita, ed. M. Gorra, Torino 1954, 13. A. Condivi, Vita di Michelagnolo Buonarroti, ed. G. Nencioni, Firenze 1998, 9. Vasari, Le vite, ed. Milanesi (nt. 15), vol. 7, 13 sq.

29 Shearman, Raphael in Early Modern Sources (nt. 7), vol. 1, 180-183. 
ausführen wollte ${ }^{30}$. Am Ende war er nach seiner eigenen Darstellung trotzdem der Klügere. Er rette den Guss, den seine Helfer fast zunichte gemacht hätten. Die alten Meister lobten ihn dafür. Abschließend kommt Cellini zu dem Fazit: ,IIch wusste wohl, dass ich den größten Teil dessen, was sie an mir lobten, von ihnen gelernt hatte. Sie folgten einer ununterbrochenen Tradition, ich aber lernte aus ihrer Erfahrung und leitete daraus die ihr zugrunde liegenden Gesetzmäßigkeiten ab“. Deshalb war er am Ende der Klügere.

Insgesamt, sollte sich abzeichnen, war die Ausbildung der Künstler zu Beginn der Neuzeit in Italien kaum geregelt, und ausgerechnet für die verantwortungsvollste Sparte, die Architektur, gab es überhaupt keine spezielle Ausbildung. Diese Situation wirkt geradezu, als hätten damals Blinde den Baubetrieb geleitet. Jesus hat das Sprichwort geprägt: „Wenn ein Blinder den anderen führt, dann fallen sie beide in die Grube" (Mat. 15, 14). Pieter Brueghel hat das in einem Bild dargestellt (1568). Aber diese Binsenweisheit galt offenbar nicht für italienische Architekten. Die italienische Architektur erreichte ja in der Renaissance höchstes Niveau und wurde zum Vorbild für viele andere Länder, wie Deutschland und Frankreich, wo die architektonische Ausbildung sauber geregelt war mit festem Pensum, Prüfungen etc. Im Lauf der Renaissance verbreitete sich sogar die italienische Praktik, Architekten ohne einschlägige Ausbildung anzustellen. In Italien bemerkte man die Diskrepanz zwischen mangelnder fachlicher Ausbildung und hohem fachlichen Anspruch in diversen Gebieten, nicht nur in der Architektur. Obwohl Alberti keinerlei handwerkliche Ausbildung hatte, verfasste er auch ein Traktat über die, Elemente der Malerei' (wohl um 1435). $E_{s}$ ist die erste Schrift ihrer Art in der Renaissance. Er leitet sie mit der Frage ein: „Hast du je einen Blinden gesehen, der einem Sehenden den Weg gewiesen hätte? Mit diesen kurzen Aufzeichnungen, die wir Elemente nennen, wirst du dich bei uns überzeugen, dass einer, der selbst vielleicht nicht zeichnen kann, doch das wahre Verfahren aufzeigt, um ein vollkommener Zeichner zu werden" 31 .

Damit kommen wir zu dem, was die Qualifikation der Architekten zu Beginn der Neuzeit eigentlich ausmachte. Manetti fasst zusammen, was Brunelleschi trotz seiner geringen Erfahrung im Bauwesen dafür qualifizierte, den Bau der Kuppel des Florentiner Doms erfolgreich durchzuführen: So eine großartige Leistung könne nicht mit dem bekannten Sachwissen gemeistert werden, schreibt er. Dazu brauche es Persönlichkeiten von hohem geistigen Niveau, voll Von Umsicht und von guten Ideen, frei von Dummheit, Schwachheit und Dünkel. Die Voraussetzung dafür, dass sich Brunelleschi überhaupt für den Bau der Kuppel engagierte, sei sein Vertrauen darein gewesen, sagt Manetti, dass es ,auf irgendeine Weise" gelingen werde, das zu vollenden, was nötig war ${ }^{32}$.

\footnotetext{
${ }^{30}$ B. Cellini, I trattati dell'oreficeria e della scultura, 3 (Trattato della scultura), ed. L. De-Mauri, Milano 1927, 203, 210.

${ }^{31}$ L. B. Alberti, Das Standbild. Die Malkunst. Grundlagen der Malerei, edd. O. Bätschmann/

C. Schäublin, Darmstadt 2000, 367, cf. 336 sq.
Manetti, Vita di Filippo Brunelleschi, edd. de Robertis/Tanturlis (nt. 5), 57.
} 
Brunelleschi trat durch eben das hervor, was seinerzeit in der Erziehung und Ausbildung neben der Lehre des reinen Sachwissens gefördert werden sollte: Weitblick, Kreativität und Eigeninitiative, Risikobereitschaft und Mut zum „Learning by doing“, wie man neudeutsch sagt. Aufgrund dieser Qualitäten schaffte er es nicht nur ,auf irgendeine Weise“, die Domkuppel zu realisieren, sondern darüber hinaus die neuzeitliche Architektur zu begründen.

Was die herausragenden Architekten der Renaissance generell auszeichnete, war die geistige Wendigkeit und der Mut, Neues zu kreieren. Das hatten sie den Handwerkern voraus, die ihre Anweisungen ausführten. Die Handwerker folgten dem, was durch Gewohnheit erprobt war, wie Cellini, Scamozzi ${ }^{33}$ und andere schreiben. Gleich zu Beginn der Renaissance erfasste der Sieneser Ingenieur Mariano Taccola generell für sein Gewerbe, was Cellini beim Bronzeguss angeblich real erlebte: nämlich dass der Architekt über Wendigkeit und Tatkraft verfügen sollte. Nur so, nicht durch tradiertes Wissen, sei er in der Lage, die vielen unvorhergesehenen Dinge zu bewältigen, die geschähen ${ }^{34}$. Manetti meint, mit angelerntem Wissen könne man nicht zur wahren Meisterschaft gelangen. Natürliches Talent und persönlicher Einsatz seien nötig, um neue „Erfindungen“ (,invenzioni") zu machen. Leonardo da Vinci hat die Methode seiner Studien durch die Gegenüberstellung von tradiertem Wissen bzw. dem üblichen Glauben und selbstständiger Erfahrung auch im Wissenschaftsbetrieb charakterisiert:

„Viele glauben mir vorwerfen zu können, dass meine Beweisführung der Autorität von hochverehrten Leuten entgegensteht $[\ldots]$, aber sie bedenken nicht, dass meine Sachen hervorgegangen sind aus der einfachen und reinen Erfahrung, die die wahre Meisterin ist. Auf diese Weise kann man das Wahre vom Falschen unterscheiden, und so nimmt man sich mit Bescheidenheit vor, was möglich ist, statt in Ignoranz gehüllt an dem zu scheitern, was ineffizient ist." 35

So dachten damals viele. Der eigenständige innovative Geist, der da kultiviert wurde, unterschied die avantgardistischen Architekten von den üblichen Bauhandwerkern, die nur das fortsetzten, was durch Erfahrung bekannt war. Er bildete zusammen mit dem futuristischen Glauben an die Zukunft den Motor für den schnellen Fortschritt an der Wende zur Neuzeit. Hier, wie damals meist, lag das Geheimnis des Erfolgs, die Kraft, die die gesamte Bewegung der Renaissance antrieb, in Eigeninitiative und Kreativität statt in Reglementierung.

Auch die von Manetti oder Leonardo herausgestellte Freiheit von Dünkel oder Bescheidenheit war wichtig. Sie wirkte sich darin aus, dass die Architekten offen waren für Experimente und für Rat. Sie ermöglichte, was oben für Architekten kurz angesprochen wurde, dass Wissenschaftler und Handwerker mit dem Willen zum Experimentieren neuerdings aufeinander zugingen, und dieses Phänomen trug wesentlich zum Erfolg der Renaissance im Ganzen bei. Die

33 Scamozzi, Idea (nt. 8), 86-88.

${ }^{34}$ M. Taccola and his Book „De ingeniis“, edd. F. D. Prager/G. Scaglia, Cambridge, MA - London 1972, 98.

${ }^{35}$ Leonardo da Vinci, Milano, Biblioteca Ambrosiana, Codex Atlanticus, $119^{\mathrm{v}}$. 
Geisteshaltung, die dahinter stand, zeichnete sich auch im Schulwesen ab. Ein reales Beispiel dafür bildet die Eliteschule des Vittorino da Feltre in Mantua, in der fürstlicher Nachwuchs zusammen mit Kindern aus einfachen Verhältnissen erzogen wurde. Ein theoretisches Beispiel bildet die Gesamtschule, die Filarete zusammen mit einem Höfling seines Herzogs entworfen hat ${ }^{36}$. Hier sollten alle Arten von Ausbildung, von der Grundschule über Werkstätten bis hin zur akademischen Qualifikation, unter einem Dach vereinigt sein, und die Jugendlichen durften im Lauf der Ausbildung je nach Talent und Neigung entscheiden, welche Laufbahn sie einschlagen wollten.

${ }^{36}$ Filarete, Trattato di architettura, edd. Finoli/Grassi (nt. 8), 493-528. H. Günther, Utopische Elemente in Filaretes Idealstadt Plusiapolis, in: A. Dietl/W. Schöller/D. Steuernagel (eds.), Utopie, Fiktion, Planung. Stadtentwürfe zwischen Antike und früher Neuzeit, Regensburg 2014, 197-220. 